\title{
Magnetoelastic effects and magnetization plateaus in two-dimensional systems
}

\author{
Samuele Bissola, ${ }^{1}$ Valeria Lante, ${ }^{1}$ Alberto Parola, ${ }_{1}^{1}$ and Federico Becca ${ }^{2}$ \\ ${ }^{1}$ Dipartimento di Fisica e Matematica, Università dell'Insubria, Via Valleggio 11, I-22100 Como, Italy \\ ${ }^{2}$ CNR-INFM Democritos National Simulation Center and International School for Advanced Studies (SISSA), \\ via Beirut 2-4, I-34014 Trieste, Italy
}

(Received 14 March 2007; published 31 May 2007)

\begin{abstract}
We show the importance of both strong frustration and spin-lattice coupling for the stabilization of magnetization plateaus in translationally invariant two-dimensional systems. We consider a frustrated spin-1/2 Heisenberg model coupled to adiabatic phonons under an external magnetic field. At zero magnetization, simple structures with two or at most four spins per unit cell are stabilized, forming dimers or $2 \times 2$ plaquettes, respectively. A much richer scenario is found in the case of magnetization $m=1 / 2$, where larger unit cells are formed with nontrivial spin textures and an analogy with the corresponding classical Ising model is detectable. Specific predictions on lattice distortions and local spin values can be directly measured by $\mathrm{x}$ rays and nuclear magnetic resonance experiments.
\end{abstract}

DOI: 10.1103/PhysRevB.75.184444

\section{INTRODUCTION}

A magnetization plateau occurs when the magnetization remains constant over a range $\Delta h$ of applied magnetic fields. The width of the plateau can be expressed in term of the excitation spectrum $\Delta h=E(M+1)-2 E(M)+E(M-1)$, where $E(M)$ is the total energy at fixed magnetization $M$ (measured in units of $g \mu_{B}$ ). This, in turn, implies that the energy (per site) as a function of the magnetization (per site) displays a cusplike singularity in the thermodynamic limit. In general, plateaus are absent in classical models with magnetic ground states whenever the magnetization is not collinear with the field. ${ }^{1}$ Instead, they identify particularly stable quantum phases characterized by a spin gap.

Usually, gaps in the excitation spectrum directly reflect the structure of the primitive cell of the lattice according to the commensurability condition ${ }^{2}$

$$
l S(1-m)=\text { integer }
$$

where $S$ is the magnitude of the spin, $m$ the magnetization per site in units of $g \mu_{B} S$ (i.e., the average on-site spin component parallel to the magnetic field), and $l$ the number of spins in the primitive cell. According to Eq. (1), magnetization plateaus in models with a single spin-1/2 per unit cell may occur only as a consequence of the spontaneous breaking of the translational symmetry, leading to an $l$-fold degenerate ground state. Although this relation has been rigorously proved in one-dimensional (1D) systems, ${ }^{2}$ it is believed to have a much wider validity. ${ }^{3}$ Several analytical and numerical calculations in chains and ladders have definitively confirmed the presence of various magnetization plateaus at the expected positions. ${ }^{4-6}$ Moreover, few important cases of plateaus in higher dimensions are also known. The most relevant example is given by the orthogonal dimer Heisenberg model, which closely represents the structure of copper planes in $\mathrm{SrCu}_{2}\left(\mathrm{BO}_{3}\right)_{2}{ }^{7}$ In this case, the theoretical predictions have been confirmed by experimental evidence of magnetization plateaus at $m=0,1 / 8$, and $1 / 4 .{ }^{8}$ Moreover, also the plateau at $m=1 / 3$ has been theoretically proposed ${ }^{9}$ and experimentally found. ${ }^{10}$ Some evidence for a $m=1 / 3$ plateau has been also proposed for the triangular lattice, ${ }^{11,12}$ while in
PACS number(s): 75.10.Jm, 71.27.+a, 74.20.Mn

the square lattice, the $m=0$ properties of the $J_{1}-J_{2}$ model are still debated. Indeed, although for $J_{2} / J_{1} \sim 1 / 2$ the ground state is believed to be disordered, the existence of a finite triplet gap is much less clear, ${ }^{13,14}$ casting some doubt on the possibility to stabilize a magnetization plateau for $m=0$.

The interest in the $J_{1}-J_{2}$ model has grown due to the recent discovery of two materials well described by a twodimensional (2D) frustrated quantum antiferromagnet, i.e., $\mathrm{Li}_{2} \mathrm{VOSiO}_{4}$ and $\mathrm{VOMoO}_{4} \cdot{ }^{15,16}$ Although the experimental magnetization curve of these compounds has not yet been considered, the magnetization properties of the $J_{1}-J_{2}$ model have been recently examined by using exact diagonalization calculations, leading to some evidence in favor of a plateau at $m=1 / 2 .{ }^{17}$ This outcome has been interpreted as a consequence of the emergence of a $2 \times 2$ supercell. According to this scenario, inside each cell the magnetic moments acquire a preferential orientation along the direction of the magnetic field, leading to a configuration with three up and one down spin. ${ }^{17}$ Unfortunately, the presence of such a halfmagnetization plateau is limited to a very narrow region close to $J_{2} / J_{1} \sim 1 / 2$, revealing the difficulty of stabilizing such a state in the pure spin model without other degrees of freedom. In this respect, the spin-lattice coupling represents one of the most relevant physical mechanisms to enhance the stability of phases breaking some of the lattice symmetries, making it easier to identify these states in numerical studies of small lattices. From general arguments, the superexchange couplings are ultimately generated by the virtual hopping of electrons through neighboring sites and strongly depend upon lattice distortions. ${ }^{18}$ The role of the spin-lattice coupling in frustrated spin systems has been extensively considered in the absence of an external magnetic field for both 1D (Refs. 19 and 20) and 2D systems. ${ }^{21}$ More recently, the importance of lattice distortions for stabilizing magnetization plateaus has been discussed in a simple 1D spin model, ${ }^{22}$ and in a classical Heisenberg model on a pyrochlore lattice. ${ }^{23}$

In this work we investigate the possible occurrence of magnetization plateaus in the frustrated spin-1/2 Heisenberg model on the square lattice by the numerical analysis of the periodicity of the distortion pattern induced by spin-lattice coupling. The paper is organized as follows. In Sec. II, we 
introduce the model, in Sec. III we present our results, and in Sec. IV we draw the conclusions.

\section{MAGNETOELASTIC EFFECTS AND MAGNETIZATION PLATEAUS}

The model is defined by

$$
\mathcal{H}=\frac{1}{2} \sum_{i, j} J\left(d_{i j}\right) \mathbf{S}_{i} \cdot \mathbf{S}_{j}+\frac{1}{2} \sum_{i, j} \frac{K\left(d_{i j}^{0}\right)}{2}\left(\frac{\left\|\delta \mathbf{r}_{i}-\delta \mathbf{r}_{j}\right\|}{d_{i j}^{0}}\right)^{2},
$$

where $\mathbf{S}_{i}$ is the spin-1/2 operator at site $i, \delta \mathbf{r}_{i}$ is the displacement of atom $i$, assumed to be in the plane, and $d_{i j} \equiv \| \mathbf{r}_{i}$ $-\mathbf{r}_{j} \|$ is the distance between atoms $i$ and $j$. The sum runs over nearest [i.e., $\left.J_{1}=J(1)\right]$, second [i.e., $\left.J_{2}=J(\sqrt{2})\right]$, and third [i.e., $\left.J_{3}=J(2)\right]$ neighbor sites on a $2 \mathrm{D}$ square lattice, while $d_{i j}^{0}=\left\|\mathbf{R}_{i}^{0}-\mathbf{R}_{j}^{0}\right\|$ is the distance between sites $i$ and $j$ in the undistorted lattice. Energy units are fixed by the choice $J_{1}=1$. In order to limit the number of parameters, only the elastic constant between nearest-neighbor sites $[K=K(1)]$ has been included. However, we checked that our conclusions are not qualitatively affected by considering further neighbor elastic constants. In transition metal compounds, the super-exchange theory combined with empirical dependence of hopping integrals on distance ${ }^{18}$ leads to exchange integrals that vary as the inverse of the distance to a given power $\alpha_{\mu}(\mu=1,2,3)$. For small displacements we can write

$$
J\left(d_{i j}\right)=J\left(d_{i j}^{0}\right)\left(\frac{d_{i j}^{0}}{d_{i j}}\right)^{\alpha_{\mu}} \simeq J\left(d_{i j}^{0}\right)\left(1-\alpha_{\mu} \frac{\delta d_{i j}}{d_{i j}^{0}}\right),
$$

with $\delta d_{i j}=d_{i j}-d_{i j}^{0} \sim\left(\mathbf{R}_{i}^{0}-\mathbf{R}_{j}^{0}\right) \cdot\left(\delta \mathbf{r}_{i}-\delta \mathbf{r}_{j}\right) / d_{i j}^{0}$. Since the Hamiltonian is invariant under the rescaling $\alpha_{\mu}=\lambda \alpha_{\mu}, K$ $=\lambda^{2} K$ and $\delta \mathbf{r}_{i}=\delta \mathbf{r}_{i} / \lambda, K$ can be fixed. To study this system, we adopt the Lanczos diagonalization technique in finite clusters with periodic boundary conditions, which allows for an unbiased determination of the lowest-energy configuration at fixed distortion pattern. In order to determine the optimal configuration of lattice displacements $\delta \mathbf{r}_{i}$ we use an iterative procedure. ${ }^{21}$ Since the local displacement of each spin from its equilibrium position is considered, we are able to describe all kinds of distortions, including the dilation and the shrinking of the lattice. We performed systematic calculations at different frustrations and lattice couplings in a $4 \times 4$ cluster at $m=0$ and $m=1 / 2$, i.e., at the magnetization values where plateaus are commonly expected in this geometry.

\section{RESULTS}

\section{A. Zero magnetization}

At vanishing magnetization, the classical $J_{1}-J_{2}-J_{3}$ model on a rigid lattice shows four magnetically ordered states: Néel with momentum $(\pi, \pi)$, collinear with momenta $(\pi, 0)$, and $(0, \pi)$ and two helicoidal phases with momenta $(q, q)$ and $(q, \pi),(\pi, q)$, where $q$ varies continuously with the parameters in the Hamiltonian. ${ }^{24-26}$ Quantum fluctuations, enhanced by the competing antiferromagnetic couplings, can drive the system away from these semiclassical behaviors and stabilize unconventional quantum phases without long-
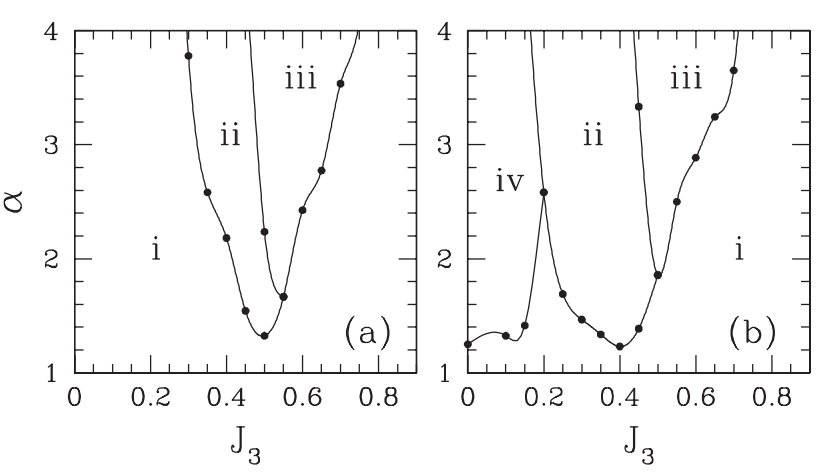

FIG. 1. Ground-state phase diagram of the $J_{1}-J_{2}-J_{3}$ model for $m=0, K=10$, and $J_{2}=0$ (a) or $J_{2}=0.5$ (b). See text for the precise description of the various phases.

range magnetic order, which may eventually display a magnetization plateau. At $J_{3}=0$, the magnetically disordered regime is widely believed to occur around the maximally frustrated point (i.e., $J_{2}=1 / 2$ ), while at $J_{2}=0$ a nonclassical phase appears between the Néel and the spiral state $(q, q)$ close to $J_{3} \sim 1 / 2$. However, in both cases, the nature of the disordered phases is still controversial and several proposals appear in the literature: ${ }^{27}$ Valence bond crystal (VBC) columnar states ${ }^{28,29}$ or spin liquids. ${ }^{13,30}$ Very recently a singlet state with plaquette order has been claimed to be stabilized around the line $J_{2}+J_{3} \simeq J_{1} / 2$ with $J_{3}>0 .{ }^{31}$

When the spin-lattice coupling is taken into account, we expect that each of the previous orderings would lead to a characteristic distortion pattern which can be easily identified. In Fig. 1 we present the results for the ground-state phase diagram obtained by Lanczos diagonalizations of the Hamiltonian (2) in the $J_{3}-\alpha$ plane for $\alpha_{\mu}=\alpha$ (for $\mu=1,2,3$ ) and $K=10$. Two values of $J_{2}=0$ and $1 / 2$, representative of the weak and strong frustration regime, will be considered.

Among all possible lattice deformations, only four are stable at $m=0$, depending on the values of the parameters (see Fig. 1). In all the states we found by numerical minimization of the ground state energy in the $4 \times 4$ cluster, the local magnetization $\left\langle S_{i}^{z}\right\rangle$ is uniform throughout the lattice. The four phases can be classified according to the different lattice distortions. (i) The square lattice with uniform bond lengths: All spatial symmetries are preserved and the presence of the spin-phonon coupling $\alpha$ just leads to a renormalization of the bond lengths. The absence of distortions for low values of $J_{3}$ is consistent with a magnetic ground state displaying Néel order at $J_{2}=0$. (ii) The dimerized lattice with two different bonds in one direction and one bond in the other, for intermediate values of $J_{3}$ and for both $J_{2}=0$ and $1 / 2$. This phase breaks both $\pi / 2$ rotation and the translational symmetry along one direction and the ground state is fourfold degenerate. In this case, the commensurability condition (1) is satisfied leading to a spin gap and a magnetization plateau. (iii) The square-plaquette phase, with dimerization in both directions for larger $J_{3}$. In this state, rotational symmetry is preserved since a $2 \times 2$ primitive cell is stabilized. Also in this case the ground state is fourfold degenerate and the spin gap is finite. In any case, the plausible occurrence of spiral magnetic ordering with incommensurate $q$ 


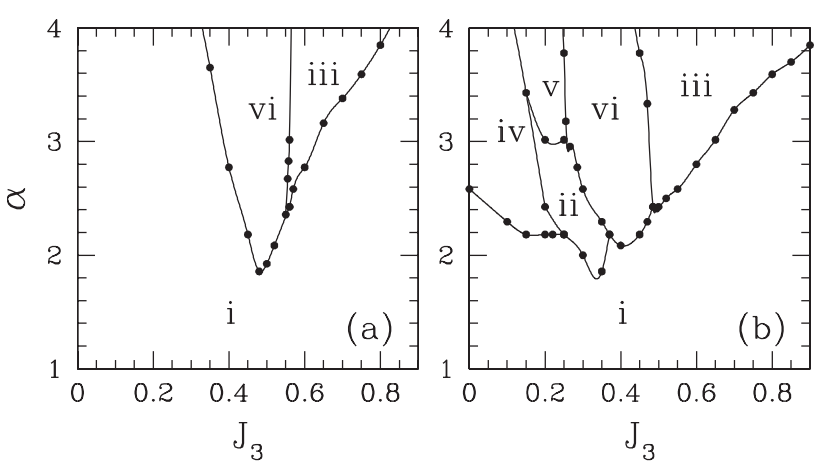

FIG. 2. The same as in Fig. 1 but for $m=1 / 2$.

at large $J_{3}$ suggests to be cautious with the numerical results in small lattices for $J_{3} \gtrsim 0.6$. For $J_{2}=1 / 2$, we also found (iv) the rectangular phase with different bond lengths in the $x$ and $y$ directions at small enough $J_{3}$. This phase breaks the $\pi / 2$ rotational symmetry but is translationally invariant, as expected when collinear magnetic ordering is present, leading to a twofold degenerate ground state: A finite-temperature phase transition marks the onset of collinear order. ${ }^{32}$ Whenever the spatial symmetry breaking persists in the absence of phonon coupling, the threshold value of $\alpha=0$ which defines the lower transition line is expected to collapse to zero as the system size is increased.

In order to better understand the physical properties of the two intermediate phases (ii) and (iii) we have analyzed the energy spectrum both in the $4 \times 4$ and in the $6 \times 6$ rigid lattice. Some evidence of translational symmetry breaking emerges from the quasi degeneracy of the states with momenta $(0,0),(0, \pi)$, and $(\pi, 0)$. However, the ordering of energy levels on these small systems is not able to discriminate between a columnar dimer or a plaquette state, because both the $d$-wave, zero momentum state, and the $(\pi, \pi)$ $s$-wave singlet have comparable energies in these clusters.

\section{B. One-half magnetization}

Let us now move to the more interesting case of $m=1 / 2$. The phase diagram is shown in Fig. 2. At small $J_{3}$, a semiclassical scenario is consistent with both the shape of the lattice and the observed uniform local magnetization, i.e., $\left\langle S_{i}^{z}\right\rangle=1 / 4$. Indeed, an applied magnetic field cants the spins which preserve a magnetic ordering in the plane orthogonal to the field direction. The numerical evaluation of the spinspin correlation function $S(k)$, by means of Lanczos diagonalizations on the $4 \times 4$ and $6 \times 6$ rigid cluster is fully compatible with the semiclassical scenario both at small and large $J_{2}$, namely, weak correlations along the magnetic field and marked peaks of the structure factor in the orthogonal plane, see Fig. 3. The analytical evaluation of the susceptibility via spin-wave theory shows that, for weak phonon coupling, the lattice undergoes global deformations but bond lengths and local magnetizations remain uniform throughout the lattice, thereby inhibiting magnetization plateaus.

By increasing the third neighbor coupling $J_{3}$, in addition to the same lattice deformations already found at zero magnetization, other two phases emerge (see Fig. 2): (v) For $J_{2}$ $=0.5$ and $\alpha$ large enough, the "trapezoidal" phase characterized by a $4 \times 2$ primitive cell made of two aligned congruent isosceles trapezia, rotated by $\pi$ one respect to the other (see Fig. 4). In this case, the lattice dilates in one direction and exhibits three different bond lengths, still preserving a rectangular shape. The local magnetization is not uniform but it acquires two different values: One is close to zero, while the other approaches the limiting value $S=1 / 2$ (see Fig. 5). (vi) The "classical Ising" phase characterized by a $4 \times 4$ unitary cell composed of four identical scalene trapezia which can be obtained one from the other by means of suitable rotations (see Fig. 4). The lattice is not squeezed in any direction, but develops a complex bond pattern with eight different bond lengths and three values of the local magnetization: Two
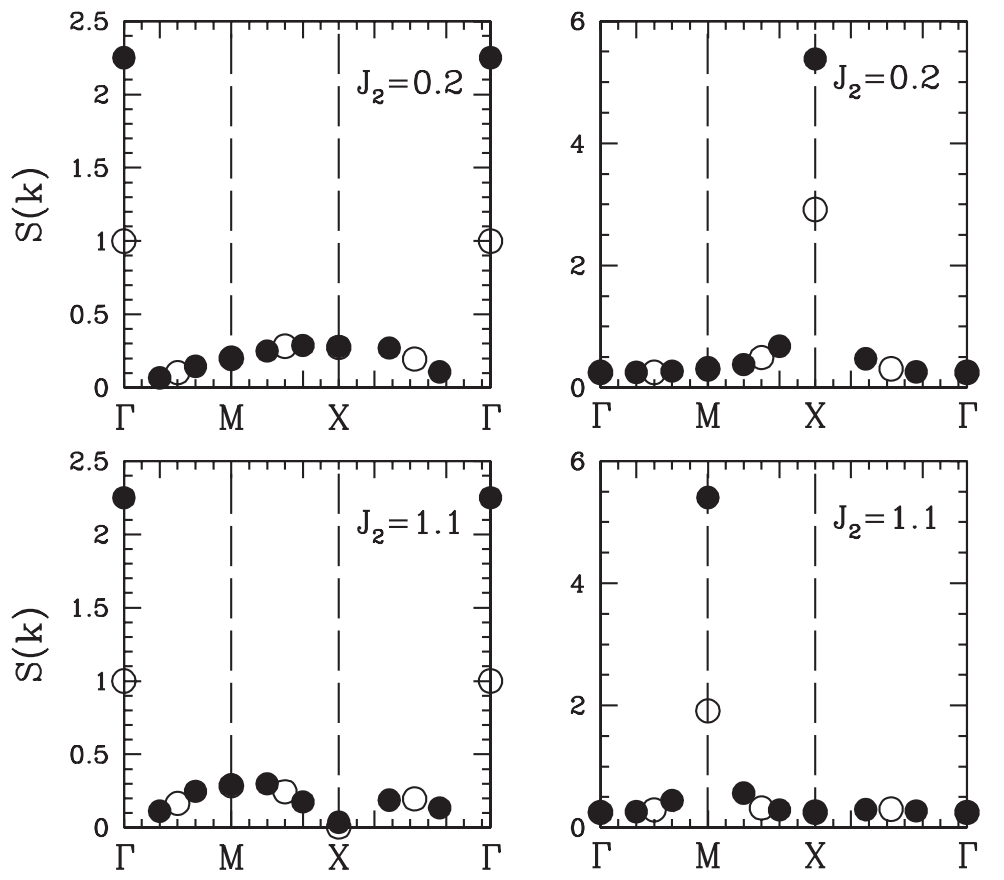

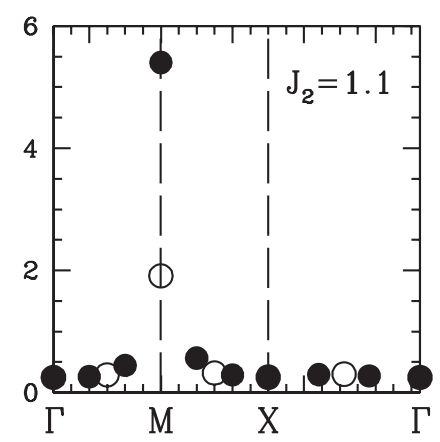

FIG. 3. Magnetic structure factor at $m=1 / 2$ along a closed path in the Brillouin zone $[\Gamma$ $=(0,0), M=(0, \pi)$, and $X=(\pi, \pi)]$ for the $J_{1}-J_{2}$ Heisenberg model $\left(J_{3}=0\right)$ on a $4 \times 4$ (empty dots) and a $6 \times 6$ (full dots) rigid lattice along the magnetic field (left panels) and in the orthogonal plane (right panels) for $J_{2}=0.2$ in the upper panels and $J_{2}=1.1$ in the lower ones. 

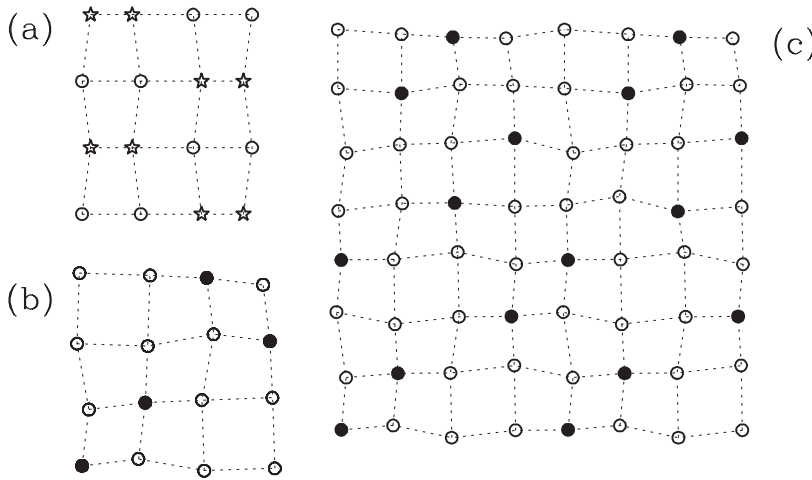

FIG. 4. Distortion pattern of the $J_{1}-J_{2}-J_{3}$ Heisenberg model on the $4 \times 4$ lattice at $m=1 / 2$ in the "trapezoidal" phase at $J_{2}=0.5$, $J_{3}=0.2$, and $\alpha=3.5$ (a) and in the "classical Ising" phase at $J_{2}$ $=0.5, J_{3}=0.4$, and $\alpha=\sqrt{10}$ (b). Distortion pattern of the Ising model on the $8 \times 8$ cluster at $J_{2}=0.5, J_{3}=0.4$, and $\alpha=\sqrt{10}$ (c). Stars mean $\left\langle S_{i}^{z}\right\rangle \sim 0$, while empty and full dots indicate positive and negative local magnetization close or equal to the maximal value $1 / 2$, respectively.

positive and one negative, with modulus close to $S=1 / 2$ (see Fig. 5). This rather rich texture of local magnetization $\left\langle S_{i}^{z}\right\rangle$ is stabilized for non-zero third neighbors coupling and can be directly tested by nuclear magnetic resonance (NMR) experiments.

Interestingly, these results admit a simple interpretation on the basis of a purely classical Ising model. Indeed, we checked that the complex topology of phase (vi) is shared by a 2D Ising model with antiferromagnetic interactions up to third neighbors and spin-lattice coupling. A numerical study of this model with $J_{2} \sim 0.5$ and $J_{3} \sim 0.4$ has been carried out both in the $4 \times 4$ and in the $8 \times 8$ lattices with similar results: The spin patterns are characterized by the requirement of minimizing the classical (Ising) contribution to the ground state energy in the undistorted lattice

$$
E_{0}=\frac{1}{2} \sum_{i, j} J\left(d_{i j}^{0}\right) S_{i}^{z} S_{j}^{z} .
$$

For the chosen parameters, this amounts to place down spins so to avoid first and third neighbors, while allowing a single

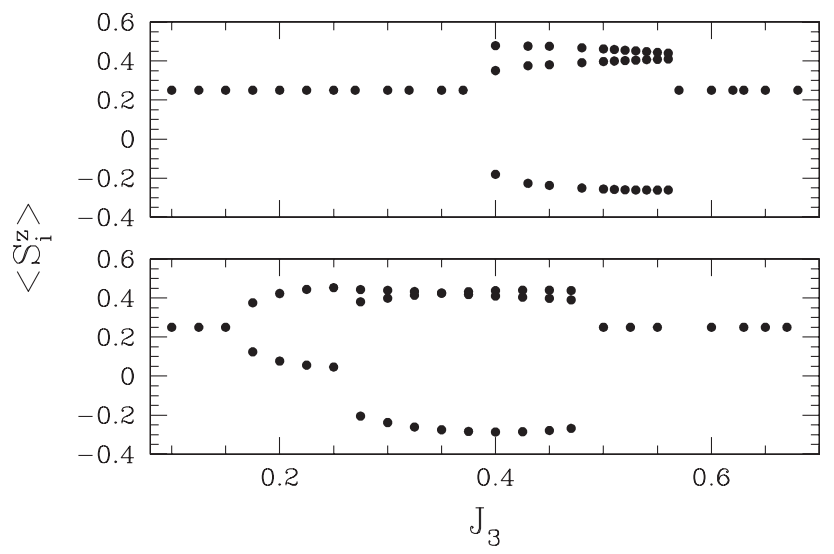

FIG. 5. Behavior of local magnetization $\left\langle S_{i}^{z}\right\rangle$ as a function of $J_{3}$ at $m=1 / 2$ and $\alpha=\sqrt{10}$ for $J_{2}=0$ (upper panel) and $J_{2}=0.5$ (lower panel). second neighbor down spin, see Fig. 4. This "topological" constraint on the spin configuration does not uniquely define the spin pattern: The resulting degeneracy is lifted by the elastic contribution, leading to tiny energy differences. ${ }^{33}$ Also the distortion pattern of the classical model is very similar to the one obtained in the quantum model (see Fig. 4), suggesting that quantum fluctuations play a minor role and finite size effects are not so relevant. It is worth noting that once the magnetic energy gain overcomes the elastic energy, the transition lines among the different phases depend only weakly on the spin-phonon coupling $\alpha$. This suggests that, in the thermodynamic limit, the distorted phases might remain stable up to arbitrarily small $\alpha$. In such a case, the $m=1 / 2$ magnetization plateau would be present, in the $J_{1}-J_{2}-J_{3}$ model, independently of the strength of spin-lattice coupling.

In order to clarify the distortion patterns obtained by a purely numerical analysis, it is instructive to discuss in more detail the case of the classical Ising model, where several results can be obtained analytically. Although the analysis may be performed in the general frustrated case, the physics emerges more clearly in the simple nearest neighbor Ising model coupled to classical phonons: The model is defined by the Hamiltonian (2) where only the $z$ component of the spin operators is retained and $J_{2}=J_{3}=K_{2}=K_{3}=0$. We denote $S_{i}^{z}$ $=1 / 2-n_{i}$, being $n_{i}$ the local density operator $\left(n_{i}=0\right.$ for a spin up and $n_{i}=1$ for a spin down), and $m=1-2 n$, being $n$ the average density. Let us fix an arbitrary spin configuration $S_{i}^{z}$ in the lattice. The total energy, as a function of the distortions $\delta \mathbf{r}_{i}$, may be minimized analytically and is given by the sum of three terms with different physical meaning: The magnetic contribution in the absence of distortions

$$
E_{0}=-\frac{J_{1} N}{2}(1-2 m)+J_{1} \sum_{\langle i, j\rangle} n_{i} n_{j},
$$

the term expressing the energy gain due to dilation

$$
E_{1}=-\frac{J_{1}^{2} N}{16 K}(1-2 m)^{2},
$$

and the highly nontrivial distortion energy

$$
E_{2}=-\frac{J_{1}^{2}}{4 K} \sum_{q \neq 0}\left|n_{q}\right|^{2} f(q),
$$

where $N$ is the number of sites, $n_{q}$ is the Fourier transform of $n_{i}$, and

$$
f(q)=\frac{\sin ^{2} q_{x}+\sin ^{2} q_{y}}{2-\cos q_{x}-\cos q_{y}} .
$$

Remarkably, these expressions may be read off as an effective Hamiltonian for the spin configuration itself: In terms of the occupation numbers $n_{i}$ this effective Hamiltonian is just

$$
\mathcal{H}_{\text {eff }}=\frac{1}{2} \sum_{i, j} v_{i j}^{\text {eff }} n_{i} n_{j}+\text { const }
$$

with effective potential given by 


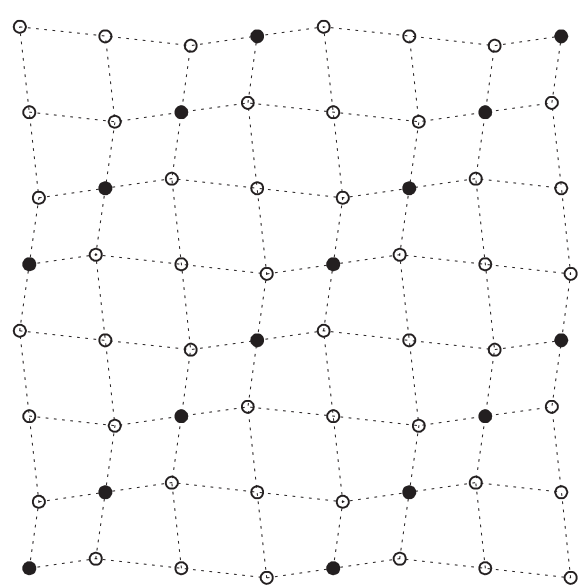

FIG. 6. Distortion pattern of the nearest-neighbor classical Ising model at $\alpha=\sqrt{10}$.

$$
v_{i j}^{\text {eff }}= \begin{cases}J_{1} & \text { if } d_{i j}^{0}=1, \\ -\frac{J_{1}^{2}}{4 K} f\left(d_{i j}^{0}\right) & \text { elsewhere }\end{cases}
$$

and $f(r)$ is the inverse Fourier transform of $f(q)$ given in Eq. (8). For $K \gg J_{1}$ the leading contribution in the effective potential is the direct nearest neighbor repulsion which selects all the configurations with no pair of nearest neighbor down spins. The contribution to the effective interaction due to lattice distortions is instead attractive for next-nearestneighbor sites and decays very quickly as a function of the distance. The most favored configuration is characterized by spins down aligned along the diagonal. In particular, for $m$ $=1 / 2$ this leads to the configuration shown in Fig. 6 and to a distortion pattern

$$
\begin{gathered}
\delta x_{i}=-\frac{J_{1}}{8 K} \sin \frac{\pi}{2}\left(x_{i}-y_{i}\right), \\
\delta y_{i}=\frac{J_{1}}{8 K} \sin \frac{\pi}{2}\left(x_{i}-y_{i}\right)
\end{gathered}
$$

which agrees with the numerical findings. Note that in this classical model, lattice distortions appear as soon as phonon coupling is switched on. Instead, no dilation is present due to the vanishing of the contribution $E_{1}$ for $m=1 / 2$.

Finally, when $J_{3}=0$ and $J_{2}$ is large enough (i.e., $J_{2} \geq 0.1$ ), the lowest-energy configuration satisfies the condition of ordering the down spins so to avoid nearest and next-nearest neighbors (see Fig. 7). This is the pattern one would also

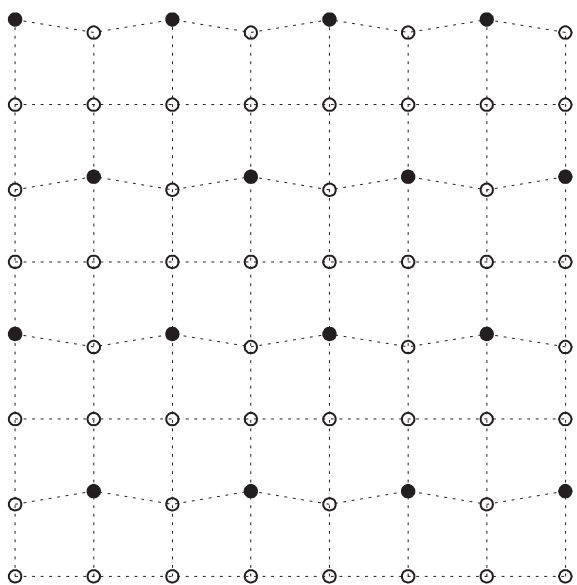

FIG. 7. Distortion pattern of the $J_{1}-J_{2}-J_{3}$ classical Ising model at $J_{2}=0.5, J_{3}=0$, and $\alpha=\sqrt{10}$.

have expected if a magnetization plateau at $m=1 / 2 \mathrm{had}$ been present in the $J_{1}-J_{2}$ Heisenberg model. However, we stress that quantum fluctuations destabilize this classical picture which appears to require the presence of spin anisotropies in the model.

\section{SUMMARY AND CONCLUSIONS}

In summary, we showed that magnetization plateaus can be stabilized in the square lattice $J_{1}-J_{2}-J_{3}$ antiferromagnetic Heisenberg model in the presence of spin-phonon coupling. Third-neighbor coupling is an essential ingredient for the appearance of plateaus both at zero and one-half magnetization. While at $m=0$ the lattice dimerizes as expected on the basis of a VBC phase, at $m=1 / 2$ a novel and complex distortion pattern characterizes the broken symmetry state. This phase can be faithfully interpreted in terms of a frustrated Ising model coupled to the lattice and appears as a genuine result not much affected by the finite size of the cluster we analyzed. The emerging scenario is considerably richer than predicted in a previous study of the $J_{1}-J_{2}$ model on a rigid lattice, ${ }^{17}$ and the on-site magnetization displays a distinctive pattern which may be measured by NMR experiments in strongly frustrated 2D magnetic materials, such as $\mathrm{Li}_{2} \mathrm{VOSiO}_{4}$ or $\mathrm{VOMoO}_{4}$.

\section{ACKNOWLEDGMENTS}

We acknowledge interesting discussion with P. Carretta and D. Poilblanc. This work has been partially supported by COFIN 2005 and CNR-INFM.
${ }^{1}$ C. Lhuillier and G. Misguich, High Magnetic Fields, Vol. 595 of Springer Lecture Notes in Physics, edited by C. Berthier, L. P. Lévy, and G. Martinez (Springer, Berlin, 2002), p. 161.

${ }^{2}$ M. Oshikawa, M. Yamanaka, and I. Affleck, Phys. Rev. Lett. 78, 1984 (1997)
${ }^{3}$ M. Oshikawa, Phys. Rev. Lett. 84, 1535 (2000).

${ }^{4}$ K. Okunishi and T. Tonegawa, J. Phys. Soc. Jpn. 72, 479 (2003).

${ }^{5}$ D. C. Cabra, A. Honecker, and P. Pujol, Phys. Rev. Lett. 79, 5126 (1997); Eur. Phys. J. B 13, 55 (2000).

${ }^{6}$ A. Honecker, F. Mila, and M. Troyer, Eur. Phys. J. B 15, 227 
(2000).

${ }^{7}$ S. Miyahara and K. Ueda, Phys. Rev. Lett. 82, 3701 (1999).

${ }^{8}$ H. Kageyama, K. Yoshimura, R. Stern, N. V. Mushnikov, K. Onizuka, M. Kato, K. Kosuge, C. P. Slichter, T. Goto, and Y. Ueda, Phys. Rev. Lett. 82, 3168 (1999).

${ }^{9}$ T. Momoi and K. Totsuka, Phys. Rev. B 61, 3231 (2000).

${ }^{10}$ K. Onizuka, H. Kageyama, Y. Narumi, K. Kindo, Y. Ueda, and T. Goto, J. Phys. Soc. Jpn. 69, 1016 (2000).

${ }^{11}$ H. Nishimori and S. Miyashita, J. Phys. Soc. Jpn. 55, 4448 (1986); A. Honecker, J. Phys.: Condens. Matter 11, 4697 (1999)

${ }^{12}$ T. Ono, H. Tanaka, H. Aruga Katori, F. Ishikawa, H. Mitamura, and T. Goto, Phys. Rev. B 67, 104431 (2003).

${ }^{13}$ L. Capriotti, F. Becca, A. Parola, and S. Sorella, Phys. Rev. Lett. 87, 097201 (2001); L. Capriotti, F. Becca, S. Sorella, and A. Parola, Phys. Rev. B 67, 172404 (2003).

${ }^{14}$ Y. Ran and X.-G. Wen, eprint arXiv:cond-mat/0609620 (unpublished).

${ }^{15}$ R. Melzi, P. Carretta, A. Lascialfari, M. Mambrini, M. Troyer, P. Millet, and F. Mila, Phys. Rev. Lett. 85, 1318 (2000); R. Melzi, S. Aldrovandi, F. Tedoldi, P. Carretta, P. Millet, and F. Mila, Phys. Rev. B 64, 024409 (2001).

${ }^{16}$ P. Carretta, N. Papinutto, C. B. Azzoni, M. C. Mozzati, E. Pavarini, S. Gonthier, and P. Millet, Phys. Rev. B 66, 094420 (2002).

${ }^{17}$ M. E. Zhitomirsky, A. Honecker, and O. A. Petrenko, Phys. Rev. Lett. 85, 3269 (2000).

${ }^{18}$ W. A. Harrison, Electronic Structure and the Properties of Solids (Dover, New York, 1980).
${ }^{19}$ J. Riera and D. Poilblanc, Phys. Rev. B 59, 2667 (1999).

${ }^{20}$ F. Becca, F. Mila, and D. Poilblanc, Phys. Rev. Lett. 91, 067202 (2003).

${ }^{21}$ F. Becca and F. Mila, Phys. Rev. Lett. 89, 037204 (2002)

${ }^{22}$ T. Vekua, D. C. Cabra, A. Dobry, C. Gazza, and D. Poilblanc, Phys. Rev. Lett. 96, 117205 (2006).

${ }^{23}$ K. Penc, N. Shannon, and H. Shiba, Phys. Rev. Lett. 93, 197203 (2004).

${ }^{24}$ A. Moreo, E. Dagotto, T. Jolicoeur, and J. Riera, Phys. Rev. B 42, $6283(1990)$

${ }^{25}$ A. Chubukov, Phys. Rev. B 44, 392 (1991).

${ }^{26}$ J. Ferrer, Phys. Rev. B 47, 8769 (1993).

${ }^{27}$ See, for instance, G. Misguich and C. Lhuillier in Frustrated Spin Systems, edited by H. T. Diep (World-Scientific, Singapore, 2005), and references therein.

${ }^{28}$ N. Read and S. Sachdev, Phys. Rev. Lett. 62, 1694 (1989).

${ }^{29}$ P. W. Leung and N. W. Lam, Phys. Rev. B 53, 2213 (1996).

${ }^{30}$ L. Capriotti, D. J. Scalapino, and S. R. White, Phys. Rev. Lett. 93, 177004 (2004).

${ }^{31}$ M. Mambrini, A. Läuchli, D. Poilblanc, and F. Mila, Phys. Rev. B 74, 144422 (2006).

${ }^{32}$ C. Weber, L. Capriotti, G. Misguich, F. Becca, M. Elhajal, and F. Mila, Phys. Rev. Lett. 91, 177202 (2003).

${ }^{33}$ This constraint leads to slightly different patterns in the $4 \times 4$ and the $8 \times 8$ clusters, because of periodic boundary conditions. The spin pattern found in the Ising model on a $4 \times 4$ lattice indeed reproduces the numerical results for the quantum Heisenberg model. 